\title{
Accumulation of stem sugar and its remobilisation in response to drought stress in a sweet sorghum genotype and its near-isogenic lines carrying different stay-green loci
}

\author{
T. Ghate ${ }^{1}$, S. Deshpande ${ }^{2} \&$ S. Bhargava ${ }^{1}$ \\ 1 Department of Botany, Savitribai Phule Pune University, Pune, India \\ 2 International Crops Research Institute for the Semi-Arid Tropics, Patancheru, India
}

\section{Keywords}

Drought stress; gene expression; sorghum; sugar accumulation.

\section{Correspondence}

Sujata Bhargava, Department of Botany, Savitribai Phule Pune University, Pune 411007, India.

E-mail: sujata@unipune.ac.in

\section{Editor}

W. Adams

Received: 5 October 2016; Accepted: 22 December 2016

doi:10.1111/plb.12538

\section{ABSTRACT}

- Near isogenic lines (NILs) of sweet sorghum genotype S35 into which individual stay green loci were introgressed, were used to understand the contribution of Stay green loci to stem sugar accumulation and its remobilization under drought stress exposure.

- Sugar and starch content, activities of sugar metabolism enzymes and levels of their expression were studied in the 3rd (source) leaf from panicle and the 5th (sugar storing) internode of the three lines, in irrigated plants and in plants exposed to a brief drought exposure at the panicle emergence stage. Annotation of genes in the respective Stay green loci introgressed in the NILs was carried out using bioinformatics tools.

- The leaves of NILs accumulated more photoassimilates and the internodes accumulated more sugar, as compared to the parent S35 line. Drought stress exposure led to a decrease in the starch and sugar levels in leaves of all three lines, while an increase in sugar levels was observed in internodes of the NILs. Sugar fluxes were accompanied by alterations in the activities of sugar metabolizing enzymes as well as the expression of genes related to sugar metabolism and transport.

- Remobilization of sugars from the stem internodes was apparent in the NILs when subjected to drought stress, since the peduncle, which supports the panicle, showed an increase in the sugar content, even when photoassimation in source leaves was reduced. Several genes related to carbohydrate metabolism were located in the Stay green loci, which probably contributed to variation in the parameters studied.

\section{INTRODUCTION}

India is a major sorghum-growing country in the world. There is immense diversity in this crop with respect to (i) tolerance to abiotic stress, (ii) photoperiod sensitivity, (iii) stem sugar accumulation and (iv) accumulation of biomass in the form of lignocelluloses. Breeding efforts in sorghum have led to the development of high grain yield (grain sorghum), high stem sugar yield (sweet sorghum) and high biomass yield (biomass sorghum). Trade-offs between grain versus stem sugars or biomass have been reported (Peloewetse 2012), making this crop suitable for either bioenergy or food. However there are also reports suggesting only minor physiological trade-offs among sugar, biomass and grain production under conditions of sufficient rainfall (Gutjahr et al. 2013a). Recent reports suggest the possibility of breeding for high grain and high stem sugar yield into a single cultivar (Murray et al. 2008; Kumar et al. 2010).

Sorghum is known to tolerate periods of water deficit through its ability to survive under reduced water potential (Tari et al. 2013). One of the adaptive mechanisms is through osmotic adjustment by soluble sugars accumulation (Kakani et al. 2011; Zegada-Lizarazu \& Monti 2013). There are reports that stem reserves in sorghum contribute to grain filling under drought stress conditions, during which photosynthesis is inhibited (Blum et al. 1997). However in some sorghum genotypes, the losses in grain yields and stem sugars due to drought stress were similar, indicating that the panicle and stems serve as independent sinks, both being affected by source limitation (Gutjahr et al. 2013b; Qazi et al. 2014). Hence the adaptation of sorghum to drought through mobilisation of soluble sugars appears to be genotype-dependent.

An important adaptive feature of sorghum, especially to post-flowering drought stress, is the stay-green phenotype. The stay-green sorghum genotypes show delayed senescence and are known to photosynthesise for a longer period from the grain filling to physiological maturity stages (Duncan et al. 1981). Prolonged photosynthesis has been correlated to better grain filling and stem sugar accumulation (Borrell et al. 2000). The stay-green sorghums were also reported to tolerate drought stress through modification of root architecture (Mace et al. 2012), reduced tillering and increased leaf size (Borrell et al. 2014), higher nitrogen use efficiency (van Oosterom et al. 2010) and improved water use efficiency (Vadez et al. 2011). The physiological mechanisms underlying the stay-green trait 
include osmotic adjustment (Anami et al. 2015) and alterations in hormone levels and hormone signalling pathways (Thomas \& Ougham 2014).

Although the stay-green trait was shown to have a genetic basis, its expression is influenced by environmental factors in certain genetic backgrounds (van Oosterom et al. 1996). Four stable stay-green quantitative trait loci (QTLs) have been identified in sorghum, namely Stg1, Stg2, Stg3 and Stg4, which accounted for $54 \%$ of the phenotypic variation and contributed to the trait across environments (Subudhi et al. 2000). Studies on the expression of genes present in the Stg QTLs have been carried out using NILs carrying different Stg QTLs and their combinations (Chaudhari \& Fakrudin 2016). Genes present in the QTL regions are thought to play a role in the maintenance of the stay-green phenotype.

Sweet sorghum genotypes into which Stg loci were introgressed show variation in traits like water extraction, transpiration efficiency, leaf area, tillering and grain yield, which depended on the introgressed loci as well as the genetic background (Vadez et al. 2011). Differential expression of the pyrroline 5 carboxylate synthase (P5CS) gene (located in Stg1 QTL) in a stay-green and a senescent genotype of sorghum was observed, and attributed to polymorphism in the cis-elements present in its promoter (Johnson et al. 2015).

In this paper, we compared sugar accumulation and the expression of genes related to sugar metabolism and transport in leaves and stem internodes of a sweet sorghum genotype S35 and two of its near isogenic lines (NILs) carrying different staygreen loci from the genotype BTx642. Variation in sugarrelated parameters was compared under irrigated conditions as well as in response to a brief drought stress exposure at the panicle emergence stage. Further, attempts were made to annotate the genes present in these QTLs and to identify those that could be putatively related to the physiological responses observed. The main objective was to understand the role of stay-green QTLs in stem sugar accumulation, as well as in remobilisation of stem sugars when plants are subjected to drought stress.

\section{MATERIAL AND METHODS}

Plant material and growth conditions

Moderately senescent sorghum caudatum variety S35 and two NILs, S35SG06040 (with Stg1 QTL) and S35SG06008 (with StgC QTL), were used for the experiments. Seeds of all three lines were procured from ICRISAT (International Crops Research Institute for the Semi-Arid Tropics, Patancheru, Andhra Pradesh, India). Seeds were sown in the field in two plots of six rows each separated by $1 \mathrm{~m}$ (Department of Botany, Savitribai Phule Pune University, Pune, India) during the post-rainy season on 10 December 2014. The average daily temperature was $15^{\circ} \mathrm{C}$ and average daily relative humidity was $89 \%$ during this period. Before sowing, equal quantities of farmyard manure was worked into the soil of each plot. Each line was sown in two randomly selected rows per plot. Both plots were irrigated by furrow irrigation at intervals of 7 days till the panicle emergence stage (60 DAS). Plants of all lines were $70-80 \mathrm{~cm}$ high and had nine to ten leaves at this stage. From 10 February 2015 watering was continued in one plot (control plot), while two irrigation cycles were skipped in the other plot (drought-treated plot). All parameters were measured after 14 days of exposure to drought stress (24 February 2015). At this time the panicles had reached anthesis stage. The soil moisture content at end of the experiment was $5 \%$ in the drought-treated plot while that in the irrigated plot was 23\% (Fig. S1).

\section{Gas exchange measurements and tissue harvest}

Gas exchange parameters, photosynthesis and stomatal conductance, were measured using the third leaf from the panicle (first fully expanded leaf) of control and drought-treated plants using an infra-red gas analyser (IRGA, LI-6200; LiCor, Licoln, $\mathrm{NB}$, USA). All readings were taken at $400 \mathrm{ppm} \mathrm{CO}_{2}, 40 \%$ relative humidity and $1000 \mu \mathrm{mol} \cdot \mathrm{m}^{-2} \cdot \mathrm{s}^{-1}$ PAR (photosynthetically active radiation) and $25^{\circ} \mathrm{C}$ leaf temperature. All measurements were made between 08:00 and 10:00 h. After imposition of drought stress for 14 days, the third leaf from the panicle and the fifth internode (which represents a sugar-storing internode in sweet sorghum stems (Qazi et al. 2012)) were harvested from each line. A small portion of lamina $\left(1 \mathrm{~cm}^{2}\right)$ from the central part of the leaf was immediately used for relative water content (RWC) measurement. The leaves and internodes were then frozen in liquid nitrogen and stored in $-80{ }^{\circ} \mathrm{C}$ within $30 \mathrm{~min}$ of harvest, for use in further experiments.

\section{Relative water content (RWC) measurements}

Relative water content was measured in the third leaf of both control and drought-treated plants after 14 days of drought treatment. Fresh weight (FW) of 1-cm leaf pieces was measured, after which the leaf pieces were placed in distilled water in Petri dishes at $4{ }^{\circ} \mathrm{C}$ for $24 \mathrm{~h}$. The leaf pieces were blotted lightly and turgid weight (TW) measured. Then the leaf pieces were oven-dried for $24 \mathrm{~h}$ and used for dry weight (DW) measurement. RWC was calculated using the formula: RWC $(\%)=100 *[(\mathrm{FW}-\mathrm{DW}) /(\mathrm{TW}-\mathrm{DW})]$.

\section{Chlorophyll measurements}

Chlorophyll content in the third leaf of each line was measured according to Arnon's method (Arnon 1949).

\section{Sap extraction}

Sap was extracted as given in Qazi et al. (2012). A total of $1 \mathrm{~g}$ de-rinded internode was homogenised in liquid nitrogen, the homogenate inserted into a $10 \mathrm{ml}$ syringe and sap collected by compressing the tissue with the plunger, until no further sap was expressed. The collected sap was centrifuged at $13,000 \times g$ at $4{ }^{\circ} \mathrm{C}$ for $10 \mathrm{~min}$ (Eppendorf 580R; Hamburg, Germany). The volume of sap was measured and expressed in $\mathrm{ml} \cdot \mathrm{g}^{-1} \mathrm{DW}$ of internode tissue.

\section{Reducing sugars and total sugar estimation}

A total of $100 \mathrm{mg}$ tissue (leaves, peduncle and de-rinded internodes) was homogenised in liquid nitrogen and re-suspended in $2 \mathrm{ml}$ ethanol, vortexed for $5 \mathrm{~min}$ and then centrifuged at $10,000 \times g$ for $10 \mathrm{~min}$ (Eppendorf 5804R). For estimation of total sugars, yeast invertase (Hi-media; 
$0.75 \mathrm{U} \cdot \mathrm{ml}^{-1}$ ) was added to the $0.5 \mathrm{ml}$ supernatant. Samples were incubated for $10 \mathrm{~min}$ at $25^{\circ} \mathrm{C}$ for conversion of nonreducing sugars to reducing sugars. Reducing sugars were estimated using dinitrosalicylic acid (DNSA) reagent (Miller 1959). A total of $0.5 \mathrm{ml}$ DNSA reagent was added to $0.5 \mathrm{ml}$ sample and the tubes placed in a water bath for $10 \mathrm{~min}$ at $100^{\circ} \mathrm{C}$. After cooling the tubes, absorbance was measured at $540 \mathrm{~nm}$ (Hitachi-2800; Tokyo, Japan). Reducing sugar concentration was calculated as glucose equivalents by comparing the absorbance with a standard curve of glucose. Both reducing and total sugars were estimated as mg glucose $\mathrm{g}^{-1} \mathrm{DW}$.

\section{Invertase (INV) assay}

Leaves or de-rinded internode samples (100 mg) were homogenised in liquid nitrogen and the homogenate suspended in $1 \mathrm{ml}$ extraction buffer ( $50 \mathrm{~mm} \mathrm{KPO}_{4}, \mathrm{pH} 7.5,5 \mathrm{~mm} \mathrm{MgCl}_{2}$ and $1 \mathrm{~mm}$ EDTA; Lingle \& Dunlap 1987). The tubes were centrifuged at $10,000 \times g\left(4^{\circ} \mathrm{C}\right)$ for $10 \mathrm{~min}$ (Eppendorf $\left.5804 \mathrm{R}\right)$. The supernatant was dialysed using dialysis tubing $(10 \mathrm{KD}$ cut off; Sigma-Aldrich, St. Louis, MO, USA) overnight against 11 $10 \mathrm{~mm} \mathrm{KPO}_{4}$ buffer $\left(\mathrm{pH} \mathrm{7.5)}\right.$ at $4{ }^{\circ} \mathrm{C}$. Dialysate was used for invertase assay. An aliquot of $1 \mathrm{ml}$ reaction mixture $(50 \mathrm{~mm}$ $\mathrm{KPO}_{4}$ buffer, $\mathrm{pH}$ 7.0, $80 \mathrm{~mm}$ sucrose and crude enzyme extract) was prepared for neutral invertase (INVN) activity whereas for acidic invertase (INVA) activity measurement $50 \mathrm{~mm}$ sodium acetate $(\mathrm{pH}$ 5) $80 \mathrm{~mm}$ sucrose and crude enzyme extract was used. Tubes were incubated for $30 \mathrm{~min}$ in a water bath at $30^{\circ} \mathrm{C}$. The reaction was stopped by adding $0.5 \mathrm{ml}$ DNSA. Tubes were again incubated for $10 \mathrm{~min}$ in a boiling water bath. The reducing sugars formed by invertase activity were estimated in terms of glucose formed $\mathrm{min}^{-1} \cdot \mathrm{mg}^{-1}$ protein.

\section{Sucrose synthase (SUS) and sucrose phosphate synthase (SPS) assays}

A total of $100 \mathrm{mg}$ leaf and de-rinded internode tissue was homogenised in liquid nitrogen and suspended in $1 \mathrm{ml}$ extraction buffer (50 mM HEPES/NaOH, pH 7.5, $7.5 \mathrm{~mm} \mathrm{MgCl}_{2}$, $2 \mathrm{~mm}$ EGTA, 2\% w/v polyethylene glycol, 2\% w/v polyvinylpyrrolidone and $5 \mathrm{~mm}$ dithiothreitol) as described in Hoffmann-Thoma et al. (1996). The tubes were centrifuged at $11,000 \times g$ (Eppendorf 5804R) at $4{ }^{\circ} \mathrm{C}$ for $10 \mathrm{~min}$ and supernatant dialyzed against $1110 \mathrm{~mm}$ HEPES ( $\mathrm{pH}$ 7.5). The dialysis was carried out using dialysis tubing (10KD cut off; Sigma) overnight at $4{ }^{\circ} \mathrm{C}$. This dialysed extract was used for sucrose synthase and sucrose phosphate synthase assays.

For sucrose synthase assay, $1 \mathrm{ml}$ reaction mixture contained $20 \mathrm{~mm}$ HEPES/NaOH buffer ( $\mathrm{pH} 7.5$ ), $5 \mathrm{~mm} \mathrm{MgCl}_{2}, 20 \mathrm{~mm}$ $\mathrm{KCl}, 12 \mathrm{~mm}$ fructose, $0.4 \mathrm{~mm}$ phosphoenolpyruvate, $2 \mathrm{~mm}$ uridine diphosphate (UDP)-glucose, $20 \mathrm{U}$ pyruvate kinase, $20 \mathrm{U}$ lactate dehydrogenase, $0.15 \mathrm{~mm} \mathrm{NADH}$ and the dialysed enzyme extract (Qazi et al. 2012). The decrease in absorbance of $\mathrm{NADH}$ was measured at $340 \mathrm{~nm}$. Sucrose synthase activity was calculated as $\mu \mathrm{mol} \mathrm{NADH}$ oxidised $\mathrm{min}^{-1} \cdot \mathrm{mg}^{-1}$ protein.

Sucrose phosphate synthase activity was also estimated from the crude extract prepared for measuring sucrose synthase activity. The reaction mixture was similar to that used for estimating sucrose synthase activity except that fructose was replaced with $15 \mathrm{~mm}$ fructose-6-phosphate. The activity was calculated as $\mu \mathrm{mol} \mathrm{NADH}$ oxidised $\mathrm{min}^{-1} \cdot \mathrm{mg}^{-1}$. protein.

\section{Starch assay}

A total of $100 \mathrm{mg}$ leaf and de-rinded internode tissue was used for estimating starch content (Starch Assay kit, STA20; Sigma). To remove non-starch sugars from the sample, samples were incubated at $85^{\circ} \mathrm{C}$ for $5 \mathrm{~min}$ in $80 \%$ ethanol. They were then washed twice with the same solvent mixture. Following washing, samples were processed according to the instructions given with the kit. Starch content was calculated as $\mathrm{mg} \cdot \mathrm{g}^{-1} \mathrm{DW}$.

\section{Gene expression studies}

Leaf and internode tissue from both control and drought-treated samples was homogenised in liquid nitrogen. Total RNA was extracted from $100 \mathrm{mg}$ using Trizol reagent (Sigma). cDNA was synthesised from $1 \mu \mathrm{g}$ RNA using ImPromII reverse transcriptase (Promega, Madison, WI, USA) and oligodT primers. Quantitative real time PCR was performed in $10 \mu$; reaction volume containing $0.3 \mu \mathrm{M}$ gene specific primer, $1 \mu \mathrm{l} \mathrm{cDNA}$ and FastStart Universal SYBR Green Master (Roche Molecular Diagnostics, Pleasanton, CA, USA). The PCR programme was: $95^{\circ} \mathrm{C}$ for $3 \mathrm{~min}$ followed by 40 cycles at $95^{\circ} \mathrm{C}$ for $10 \mathrm{~s}, 58^{\circ} \mathrm{C}$ for $20 \mathrm{~s}$ and $60^{\circ} \mathrm{C}$ for $20 \mathrm{~s}$ (Eppendorf realplex ${ }^{2}$ ). PCR specificity was checked with melting curve analysis and data was analysed using the $2^{-\Delta \Delta \mathrm{ct}}$ method (Livak \& Schmittgen 2001). The constitutively expressed EF1 $\alpha$ gene was used as internal control. Primers were designed for three INV genes, three SPS genes, three SUS genes and five sucrose transporter genes using Gene runner software. All the gene-specific primers are listed in Table S1.

\section{Annotation of genes present in Stg1 and StgC QTLs}

The GO annotation for 330 out of 531 genes in StgC QTL and 203 out of 342 genes in Stg1 QTL is available in the database. Transcript sequences of these genes were determined using Phytozome (https://phytozome.jgi.doe.gov/). Putative functions and annotations of these genes were determined with the UniProt database (www.uniprot.org), using rice as the reference genome. The AgriGO gene ontology tool (http://bioinf o.cau.edu.cn/agriGO/) was used to group genes into broad functional categories based on GO annotations.

\section{Statistical analysis}

Physiological parameters and enzyme activities were measured from three independent plants (biological replicates). ANOVA was carried out using studied parameters as responses and treatments within genotypes as factors at the panicle emergence stage using SYSTAT statistical software (https://systatsoftware.c $\mathrm{om} /$ ). Tukey's pair-wise comparison test was performed to evaluate statistical significance between two treatment means for each response. Means of three biological replicates, each with three technical replicates, were used for quantifying gene expression levels. Fold change in gene expression was analysed with Student's $t$-test for each pair of samples.

\section{RESULTS}

The NILs S35SG06040 and S35SG06008 contained about threefold more chlorophyll $\mathrm{g}^{-1} \mathrm{DW}$ in leaves as compared to chlorophyll levels in the parent variety S35. However, 
Table 1. Comparison of RWC, photosynthetic traits and carbohydrate content in leaves of sweet sorghum genotype S35 and two of its NILS, S35SG06008 and S35SG06040, grown under irrigated conditions (C) or subjected to drought exposure at panicle emergence stage for 14 days (D). Values represent means of three biological replicates. Different letters in each column indicate significant differences $(P<0.05)$.

\begin{tabular}{|c|c|c|c|c|c|c|c|}
\hline Genotype & $\begin{array}{l}\text { Relative water } \\
\text { content, \% }\end{array}$ & $\begin{array}{l}\text { Photosynthesis } \\
\text { rate, } \mu \mathrm{mol} \\
\mathrm{CO}_{2} \mathrm{~m}^{-2} \cdot \mathrm{s}^{-1}\end{array}$ & $\begin{array}{l}\text { Stomatal } \\
\text { conductance, mol } \\
\mathrm{H}_{2} \mathrm{O} \mathrm{m}^{-2} \cdot \mathrm{s}^{-1}\end{array}$ & $\begin{array}{l}\text { Chlorophyll, } \\
\mathrm{mg} \cdot \mathrm{g}^{-1} \text { DW }\end{array}$ & $\begin{array}{l}\text { Total sugars, } \\
\mathrm{mg} \cdot \mathrm{g}^{-1} \mathrm{DW}\end{array}$ & $\begin{array}{l}\text { Reducing sugars, } \\
\mathrm{mg} \cdot \mathrm{g}^{-1} \mathrm{DW}\end{array}$ & $\begin{array}{l}\text { Starch, } \mathrm{mg} \cdot \mathrm{g}^{-1} \\
\text { DW }\end{array}$ \\
\hline S35 C & $87 \pm 1.6^{a}$ & $19.86 \pm 0.28^{\mathrm{a}}$ & $0.17 \pm 0.01^{a}$ & $2.45 \pm 0.60^{b}$ & $73.02 \pm 2.03^{c}$ & $34.12 \pm 1.39^{b}$ & $13.72 \pm 1.29^{c}$ \\
\hline S35 D & $57 \pm 0.6^{b}$ & $9.46 \pm 0.52^{c}$ & $0.07 \pm 0.003^{b}$ & $0.71 \pm 0.04^{d}$ & $83.05 \pm 2.95^{c}$ & $38.55 \pm 6.19^{b}$ & $21.94 \pm 1.85^{b}$ \\
\hline S35SG06008 C & $89 \pm 0.5^{a}$ & $20.47 \pm 0.89^{a}$ & $0.18 \pm 0.02^{a}$ & $6.15 \pm 0.27^{\mathrm{a}}$ & $273.65 \pm 5.22^{a}$ & $73.75 \pm 7.92^{\mathrm{a}}$ & $40.74 \pm 1.21^{a}$ \\
\hline S35SG06008 D & $62 \pm 1.9^{b}$ & $14.06 \pm 0.57^{b}$ & $0.07 \pm 0.01^{b}$ & $1.79 \pm 0.17^{c}$ & $100.57 \pm 5.92^{c}$ & $57.95 \pm 1.45^{a}$ & $19.57 \pm 0.98^{b}$ \\
\hline S35SG06040 C & $88 \pm 0.4^{a}$ & $19.57 \pm 0.33^{a}$ & $0.18 \pm 0.01^{a}$ & $6.74 \pm 0.29^{a}$ & $145.82 \pm 1.68^{b}$ & $83.93 \pm 2.73^{a}$ & $37.09 \pm 0.72^{a}$ \\
\hline S35SG06040 D & $58 \pm 1.4^{b}$ & $11.66 \pm 0.94^{c}$ & $0.06 \pm 0.01^{b}$ & $2.44 \pm 0.11^{b}$ & $129.77 \pm 0.59^{b}$ & $71.52 \pm 5.29^{a}$ & $14.13 \pm 1.20^{c}$ \\
\hline
\end{tabular}

Table 2. Comparison of sap volume and carbohydrate content in the fifth internode (columns 2-5) and peduncle (column 6) of sweet sorghum genotype S35 and two of its NILS, S35SG06008 and S35SG06040, grown under irrigated conditions (C) or subjected to drought exposure at panicle emergence stage for 14 days (D). Values represent means of three biological replicates. Different letters in each column indicate significant differences $(P<0.05)$.

\begin{tabular}{|c|c|c|c|c|c|}
\hline Genotype & $\begin{array}{l}\text { Sap volume, } \\
\mathrm{ml} \cdot \mathrm{g}^{-1} \mathrm{DW}\end{array}$ & $\begin{array}{l}\text { Total sugars, } \\
\mathrm{mg} \cdot \mathrm{g}^{-1} \mathrm{DW}\end{array}$ & $\begin{array}{l}\text { Reducing sugars, } \\
\mathrm{mg} \cdot \mathrm{g}^{-1} \mathrm{DW}\end{array}$ & $\begin{array}{l}\text { Starch, } \\
\mathrm{mg} \cdot \mathrm{g}^{-1} \mathrm{DW}\end{array}$ & $\begin{array}{l}\text { Total sugars, } \\
\mathrm{mg} \cdot \mathrm{g}^{-1} \mathrm{DW} \text { (peduncle) }\end{array}$ \\
\hline S35 C & $1.20 \pm 0.15^{c}$ & $51.90 \pm 1.33^{d}$ & $9.60 \pm 0.67^{c}$ & $94.74 \pm 4.83^{b}$ & $48.56 \pm 2.26^{d}$ \\
\hline S35 D & $0.79 \pm 0.03^{d}$ & $30.34 \pm 3.66^{d}$ & $6.51 \pm 1.34^{c}$ & $40.44 \pm 2.31^{c}$ & $28.75 \pm 3.05^{\mathrm{e}}$ \\
\hline S35SG06008 C & $2.04 \pm 0.04^{a}$ & $143.74 \pm 10.58^{c}$ & $39.69 \pm 3.41^{b}$ & $142.10 \pm 20.27^{a}$ & $95.03 \pm 9.18^{b}$ \\
\hline S35SG06008 D & $0.82 \pm 0.03^{d}$ & $351.72 \pm 8.38^{a}$ & $83.62 \pm 3.51^{a}$ & $50.60 \pm 1.81^{c}$ & $162.55 \pm 4.80^{a}$ \\
\hline S35SG06040 C & $1.73 \pm 0.01^{\mathrm{b}}$ & $120.32 \pm 9.25^{c}$ & $32.89 \pm 2.17^{b}$ & $86.39 \pm 4.80^{b}$ & $79.01 \pm 5.32^{c}$ \\
\hline S35SG06040 D & $1.27 \pm 0.05^{c}$ & $194.38 \pm 10.35^{b}$ & $55.29 \pm 3.65^{b}$ & $47.11 \pm 1.75^{c}$ & $129.85 \pm 2.7^{b}$ \\
\hline
\end{tabular}

photosynthesis rates and stomatal conductance in all three genotypes was similar. The NILs accumulated two- to four-fold more sugar and three-fold more starch in the leaves as compared to sugar and starch content in leaves of S35 (Table 1).

Drought exposure for 14 days at the panicle emergence stage led to about $40 \%$ decrease in RWC of leaves, which was accompanied by a decrease in chlorophyll content, photosynthesis rates and stomatal conductance in all three lines. While the leaves of S35 did not show any change in total sugar or starch content as compared to controls when exposed to drought, NIL S35SG06008 showed a significant decrease in sugar content and both NILs showed about 50\% decrease in starch content on drought exposure (Table 1).

Total sugar content in the fifth internode was significantly higher in both NILs as compared to that in S35 (Table 2). Additionally, the fifth internode of NIL S35SG06008 showed about $50-60 \%$ higher sap volume and $17 \%$ more starch content as compared to that in the parent variety S35 and the other NIL S35SG06040.

On exposure to drought stress, sap volume in the fifth internode of S35SG06040 decreased by 32\% on a DW basis, while the decrease in sap volume in S35 and S35SG06008 was about 1.5 -fold and 2.5-fold, respectively (Table 2). The drought stress imposed led to no change in total sugar content, but starch content decreased by $40 \%$ in the S35 internode. The NILs accumulated more sugar (on a DW basis), with S35SG06040 showing about a 40\% increase and S35SG06008 a 2.5-fold increase in sugar levels. While most of the increase in sugar levels of S35SG06008 could be attributed to the observed decrease in sap volume (2.5-fold) on exposure to drought stress, an increase in reducing sugar content was also observed in the drought-stressed plants. The two NILs also showed a 20\% (S35SG06040) and three-fold (S35SG06008) reduction in starch content as compared to their respective controls. The peduncles, which support the developing panicle, showed about 1.7 -fold increase in total sugars in response to drought stress in the NILs, but not in S35 (Table 2).

\section{Comparison of sugar metabolism enzyme activity in S35 and the two NILs under irrigated and drought conditions}

\section{Leaves}

Activity of sugar metabolising enzymes differed in the three sweet sorghum lines. The NILs had about 50\% less activity of INVA, INVN, SPS and SUS in the leaves as compared to S35 (Table $3 \mathrm{a}-\mathrm{d}$ ). Activity of INVA and INVN increased about $35 \%$ and activity of SUS and SPS increased by about $60 \%$ in leaves of S35 after drought exposure. However in the two NILs, the activity of all these enzymes increased twofold as compared to their respective controls (Table 3a-d).

\section{Fifth internodes}

The activity of most sugar metabolising enzymes in the fifth internodes were significantly higher as compared to their activity in S35, except for SUS activity in S35SG06008, which did not differ significantly from that in S35 (Table $3 \mathrm{e}-\mathrm{h}$ ). When exposed to drought stress, S35 had twofold higher INVN and $67 \%$ higher SUS activity, while NIL S35SG06008 had a three-fold increase in INVA and INVN activity and twofold increase in SPS activity. The NIL S35SG06040, on the other hand, had about $80 \%$ higher SPS activity, but 


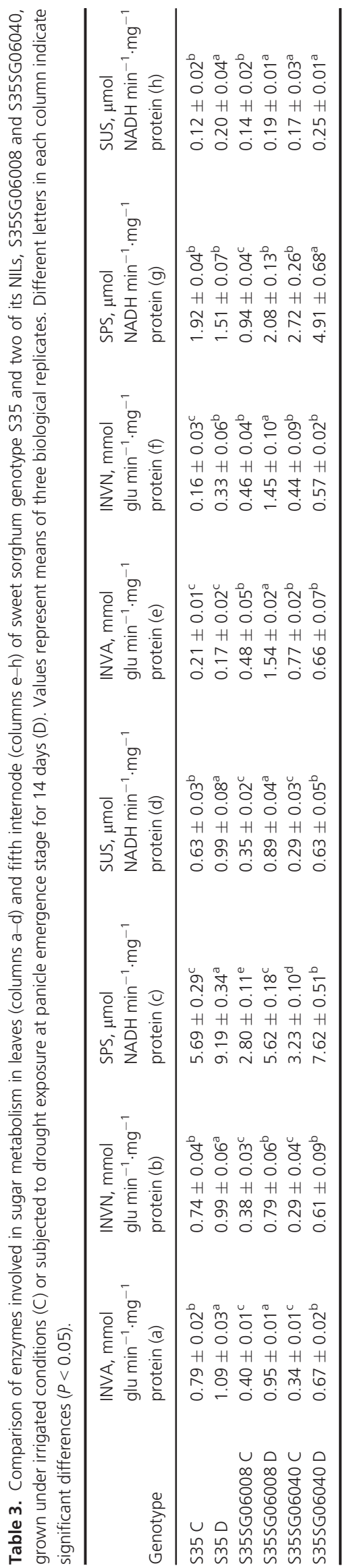

activity of the other enzymes did not alter significantly (Table $3 \mathrm{e}-\mathrm{h}$ ).

Comparison of gene expression of sugar metabolism enzymes and sucrose transporters in S35 and the two NILs under irrigated and drought conditions

Leaves

Expression of genes coding for sugar metabolising enzymes did not always correspond to activity of the enzymes that they coded for. Hence, although the NILs showed about 50\% lower activity of sugar metabolising enzymes, INV3 showed twofold higher expression in leaves of S35SG06040 (Fig. 1a), and SPS1, 2 and 3 genes had 1.5-fold higher expression in leaves of S35SG06008 as compared to their expression in leaves of S35 (Fig. 1b). Expression of SUS2 was about 50\% lower in the two NILs, while S35SG06040 also showed 50\% inhibition in SUS3 expression (Fig. 1c). Since sugar metabolism plays an important role in remobilisation of sucrose in plants, expression of sucrose transporter genes (SUTs) was studied as a proxy for their actual transporter activities. Expression of SUT genes differed in the NILs as compared to S35. Both NILs had about $50 \%$ reduced expression of SUT1 as compared to S35, while there was a 1.5-fold increase in SUT2 expression in S35SG06040 (Fig. 1d).

On drought stress exposure, leaves of both NILs showed a significant decrease in the expression of INV1 and INV3 as compared to their respective controls. Expression of SPS 1,2 and 3 was 50\% lower in NIL S35SG06008 but increased by about 1.5- to twofold in S35 and S35SG06040 (Fig. 2b). Leaves of S35SG06040 also showed a twofold increase in expression of SUS1 and SUS3, while S35SG06008 showed a 50\% decrease in expression of SUS1 and SUS2 and S35 showed a 50\% decrease in expression only of SUS2 as compared to their respective controls (Fig. 2c). Under drought exposure SUT2 showed increased expression in all three lines (Fig. 2d).

\section{Fifth internodes}

The fifth internode of S35SG06040 showed about eight- to tenfold increase in expression of both INV2 and INV3 as compared to expression in S35, while S35SG06008 only showed higher expression of INV2 (Fig. 1e). Expression of SPS3 was also 75\% higher in S35SG06040 as compared to S35, while expression of all SPS genes was about 50\% lower in S35SG06008 as compared to S35 (Fig. 1f). Among the differences in transcript abundance of the sucrose transporters, about twofold higher expression of SUT3 and SUT5 was observed in S35SG06040, while expression of SUT2 and SUT5 increased in S35SG06008, in comparison to S35. SUT1 expression decreased $50 \%$ in S35SG06040, but not in the other NIL (Fig. 1h).

On exposure to drought stress, there was a two- and four-fold increase in INV3 expression in S35 and S35SG06008, respectively (Fig. 2e). While SUS2 expression increased in all three lines in response to drought, the NILs showed about 15 -fold increase as compared to three-fold observed in S35 (Fig. 2g). Expression of genes coding for other enzymes was not significantly altered under drought. SUT2 expression in the fifth internode increased 20-fold on exposure to drought in S35SG06040, while in S35 and S35SG06008 it was about ten-fold higher. S35SG06040 also showed about three-fold higher expression of SUT1 as compared to the non-stressed controls (Fig. 2h). 

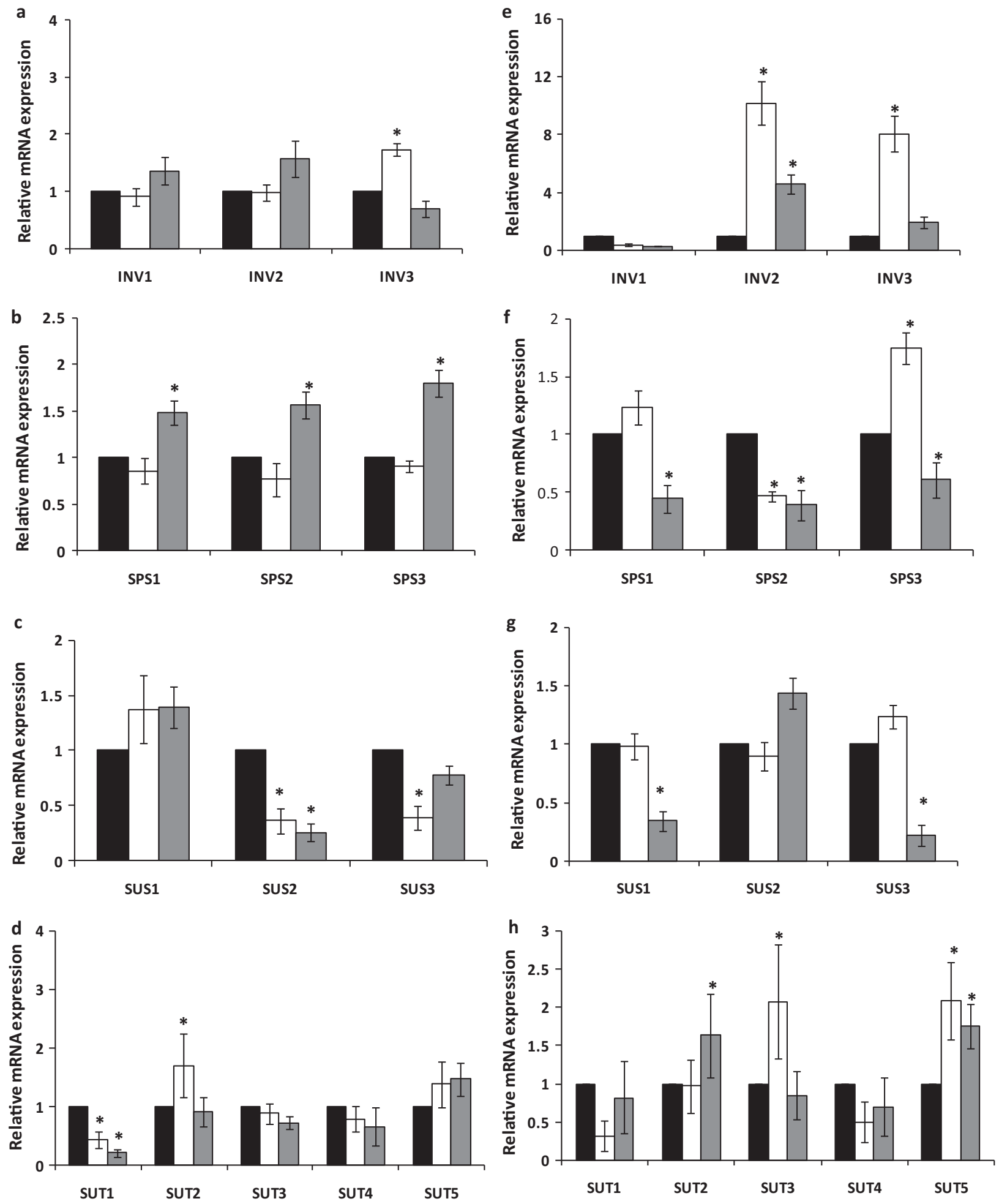

Fig. 1. Fold change in expression of genes coding for sugar metabolising enzymes and sugar transporters in leaves (a-d) and in fifth internodes (e-h) of the two NILS as compared to S35, at panicle emergence stage of irrigated plants. Black bars = S35, White bars = S35SG06040, Grey bars = S35SG06008. *Indicates significant difference in expression levels as compared to S35 $(P<0.05)$.

Analysis of gene content in QTLs Stg1 (in NIL S35SG06040) and StgC (in NIL S35SG06008)

The GO annotations were available for 330 out of 531 genes in StgC QTL and 203 out of 342 genes in Stg1 QTL in the database.
Grouping of genes into broad functional categories based on GO annotations showed a similar number of genes in categories such as catalytic activity (18-23\%), metabolic processes (21-23\%), binding (23-27\%), cellular processes (11-14\%), cellrelated $(9-10 \%)$ and biological regulation (5-7\%). Additional 

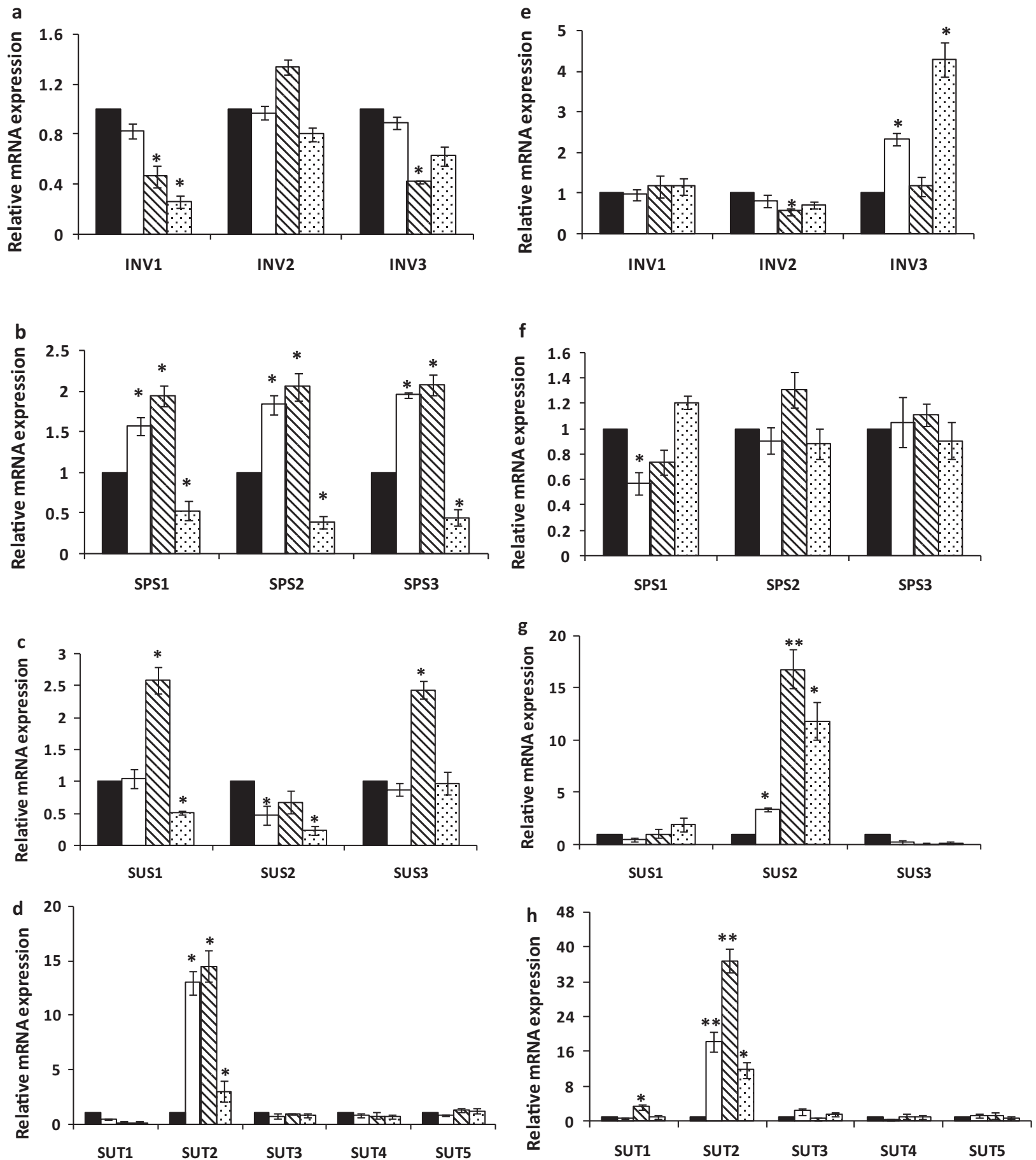

Fig. 2. Expression of genes coding for sugar metabolising enzymes and sugar transporters in leaves (a-d) and fifth internodes (e-h) in sweet sorghum plants subjected to drought stress at panicle emergence stage. Values represent fold change in expression of drought-stressed plants as compared to the respective controls (irrigated plants). Black bars $=$ control, White bars $=\$ \mathrm{~S} 35$, Dark grey bars $=\$ 355 \mathrm{SG06040}$, Light grey bars $=\$ 35 \mathrm{SG} 06008 . * P<0.05, * * P<0.01$ indicate significant differences as compared to controls.

categories such as transporter activity (2\%) and localisation (4\%) were observed in the StgC QTL (Fig. 3a,b).

Analysis of genes related to carbohydrate metabolism, which were included under the metabolic processes category of GO annotations, showed that Stg1 QTL included genes coding for glycosyl hydrolase family 17 and 18 proteins (eight genes), beta- galactosidase 3 precursor, starch/glucanphosphorylase, betaglucanase-like protein (glycosyl hydrolase family 43), fructose1,6-bisphosphatase, mannose-1-phosphate guanyltransferase and glycosyl transferase family 29. StgC QTL included genes coding for glycosyl hydrolase 17 and 18 families (four genes), UDP-glucosyltransferase, starch synthase, beta-amylase, 
a

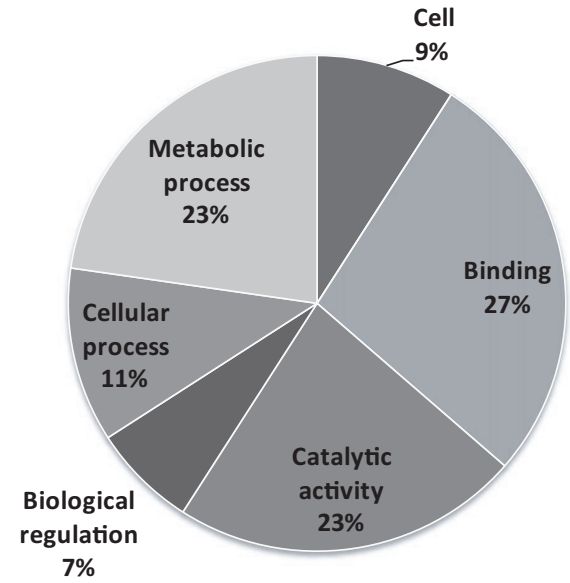

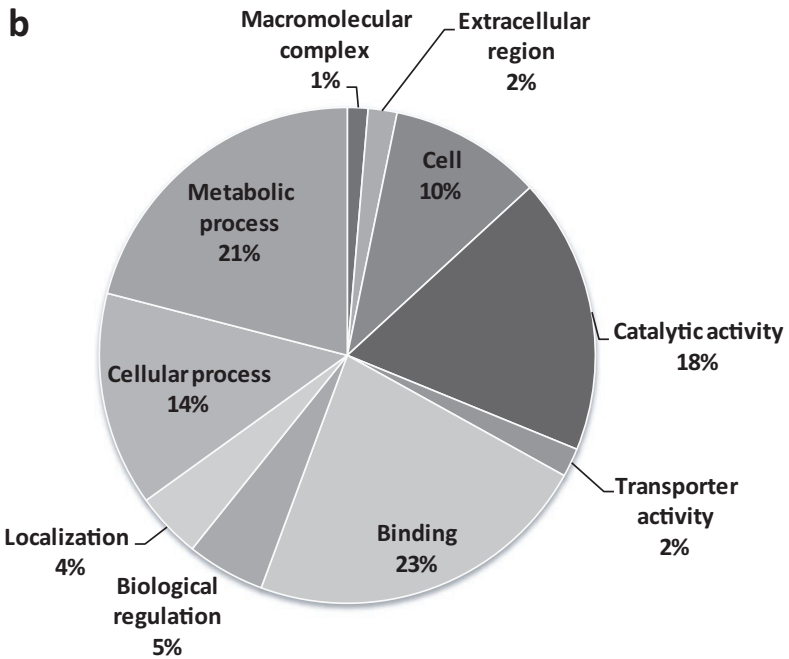

Fig. 3. Pie charts showing representation of genes on the basis of GO categories in (a) Stg1 QTL (introgressed in NIL S35SG06040) and (b) StgC QTL (introgressed in NIL S35SG06008).

glycosyl transferase family A, cellulose synthase-like family D, Invertase/pectin methylesterase inhibitor family protein, phosphofructokinase, mannan synthase 4, sucrose transporter (SUT3), phosphoglycerate kinase, pyruvate kinase and aldose 1epimerase (Table S2).

Besides carbohydrate metabolism-related genes, Stg1 QTL included stress-related genes coding for proline synthesis, ABA-induced protein phosphatase $2 \mathrm{C}$, three bZIP transcription factors and an auxin-responsive protein. StgC showed presence of genes coding for an abscisic acid-insensitive protein, dehydration response-related protein and several jasmonic acid responsive ZIM domain-containing proteins (Table S2).

\section{DISCUSSION}

The phenotypes of the two NILs differed from the parent line S35 in having higher chlorophyll, sugar and starch levels in the leaves. Although photosynthesis rates did not differ between the NILs and S35, the higher chlorophyll levels could be correlated with higher photosynthetic capacity and hence better photoassimilation. This could also explain the higher stem sugar accumulation observed in the NILs. The NILs also showed lower activity of sugar metabolising enzymes in leaves as compared to S35. Phloem loading in sorghum is thought to occur via an apoplastic pathway, which would not involve sugar inter-conversions that are necessary to maintain osmotic concentrations across membranes during symplastic transport (Tarpley \& Vietor 2007). The stem internodes of the NILs, which accumulated higher stem sugar, showed higher activity of sugar metabolising enzymes as compared to S35, indicating that sucrose storage in the sink parenchyma cells involves sucrose inter-conversions outside and within the cells, to ensure continuous sucrose flow into the sink tissues and maintain osmotic concentrations across membranes. Higher SUS and INV activity has been reported to play a role in creating a strong sink for sucrose unloading in sugarcane internodes (Schäfer et al. 2004; Grof et al. 2006). Higher expression of INV3, SUS3, SPS2 and SPS5 was observed in the internode tissue of a sweet sorghum genotype as compared to expression in a grain sorghum genotype, although activities of the sugar metabolising enzymes were not studied (Shakoor et al. 2014). Expression of genes involved in sugar transport also differed in the two NILs as compared to that in the parent line S35. For example, SUT1 expression was 50\% lower in leaves and expression of SUT5 was significantly higher in the stem internode. These transporters are known to play a role in phloem loading in source leaves and unloading in stem storage tissues, respectively (Li et al. 2014).

Although similar chlorophyll losses occurred in S35 and the NILs in response to drought stress, the NILs still had about 2.5- (S35SG06008) to 3.5- (S35SG06040) times more chlorophyll content, indicating possible better photosynthetic performance of the NILs under drought stress. However the NILs showed a significant reduction in leaf starch when subjected to drought stress. Since S35 had the lowest chlorophyll levels and yet maintained leaf starch content under drought stress, the possibility of source limitation to photoassimilation in the NILs seemed unlikely. Instead, a likely explanation was the conversion of leaf starch to sugars, which were remobilised to the stem and panicle sinks. Concomitantly, an increase in stem sugar accumulation was observed in fifth internodes and peduncles of the NILs, but not in S35. A positive correlation between stem sugar accumulation and the stay-green trait has also been reported in other stay-green sweet sorghum lines under drought conditions (Tovignan et al. 2016). Water deficit is also known to stimulate sucrose synthesis and decrease starch synthesis due to unavailability of phosphorylated metabolites (Geigenberger et al. 1997; Chen et al. 2015). When subjected to drought, the two NILs showed significantly higher activity of all sugar metabolism enzymes in leaves, accompanied by higher expression of INV1 and INV3 genes. The internodes of S35SG06040 NIL also had increased SPS activity. A positive correlation between SPS activity and rate of sucrose accumulation has also been reported in sugarcane stems (Botha \& Black 2000).

Besides the common traits observed in both NILs as compared to the parent line S35, phenotypes of the two NILs differed from each other in several respects. For example, NIL 
S35SG06008 had higher sap volume and starch content in the fifth internode than NIL S35SG06040, as well as the parent line S35. Accumulation of sugar in stems is thought to play a role in increasing osmotic pressure within cells, causing upward movement of water from soil to leaves (Fu et al. 2012), which could explain the increase in sap volume in S35SG06008. On exposure to drought stress, leaves of S35SG06008 showed a significant decrease in sugar content (which was not seen in the other NIL), correlated to lower expression of all three SPS genes and also SUS1 and SUS2 genes. The fifth internode of S35SG06008 showed a 65\% reduction in starch content on drought exposure. This NIL also had a 2.5-fold increase in sugar accumulation, which could be attributed to 2.5 -fold water loss from sap. While amylase activity was not measured, there was a three-fold increase in invertase activity and fourfold increase in expression of INV3 gene. Invertases are known as 'feast genes' that respond to high levels of sugars and bring about the feed-forward enhancement of sink strength. Higher sink demand is thought to reduce sugar levels in leaves and up-regulate expression of 'famine' genes for acquisition of limited carbon reserves (Bihmidine et al. 2015). This NIL also showed a 1.7-fold increase in sugar content of peduncles on exposure to drought. Since photoassimilation was reduced on drought exposure, the increase in sugar levels in peduncles could be explained through remobilisation of stem sugars formed from starch and sucrose breakdown in the fifth internode. The higher expression of sucrose transporters SUT2 and SUT5 seen in the stem internode of this NIL could possibly have a role in remobilising sugars from the stem to the peduncle. The NIL S35SG06040 also showed similar trends in carbohydrate metabolism during its response to drought as S35SG06008, but the magnitude of the response was lower.

Differences between sugar metabolism and accumulation in S35 and the NILs could be attributed to direct effects of genes situated in QTLs or indirect regulatory effects of these genes. On comparing the genes related to carbohydrate metabolism within these QTLs, it was seen that both QTLs contained genes related to glycosyl hydrolase families 17 and 18, which are known to play a role in starch hydrolysis and remobilisation of photoassimilates, as well as in hormone signalling through removal of sugars from hormone conjugates, besides other functions (Tyler et al. 2010). On comparing differences between the two QTL regions in these NILs, it was observed that StgC (from NIL S35SG06008) had several genes coding for glycolytic enzymes, starch synthase and a sucrose transporter, which were not observed in Stg1 (from NIL S35SG06040). Stg1, on the other hand, had about twice the number of genes related to sugar hydrolysis (glucosyl hydroxylases) as compared to StgC. Hence the two QTLs from BTx642 differed in the distribution of genes related to sugar metabolism and transport. Expression of some of the candidate genes from NILs and their parent line S35, under drought stress would be important for understanding whether they have a role in higher stem sugar accumulation, as well as in regulating sugar fluxes during exposure to drought stress, which might ensure grain filling under conditions when photoassimilation is limited.

\section{ACKNOWLEDGEMENTS}

TG and SB acknowledge financial assistance from the Department of Science and Technology, Government of India, under the PURSE program and the Board of College and University Development, Savitribai Phule Pune University, Pune.

\section{SUPPORTING INFORMATION}

Additional Supporting Information may be found online in the supporting information tab for this article:

Figure S1. Soil water content (SWC) during the period of drought stress application (14 days) in sorghum lines S35, S35SG06008 and S35SG06040. Values represent percentage of soil moisture determined from two samples per time point using the gravimetric method.

Table S1. List of primers used for gene expression studies.

Table S2. Annotation of genes located in Stg1 and StgC QTLs using rice as reference genome.

\section{REFERENCES}

Anami S.E., Zhang L.M., Xia Y., Zhang Y.M., Liu Z.Q., Jing H.C. (2015) Sweet sorghum ideotypes: genetic improvement of stress tolerance. Food and Energy Security, 4, 3-24.

Arnon D.I. (1949) Copper enzymes in isolated chloroplasts. Polyphenoloxidase in Beta vulgaris. Plant Physiology, 24, 1

Bihmidine S., Baker R.F., Hoffner C., Braun D.M. (2015) Sucrose accumulation in sweet sorghum stems occurs by apoplasmic phloem unloading and does not involve differential sucrose transporter expression. BMC Plant Biology, 15, 186.

Blum A., Golan G., Mayer J., Sinmena B. (1997) The effect of dwarfing genes on sorghum grain filling from remobilized stem reserves, under stress. Field Crops Research, 52, 43-54.

Borrell A.K., Hammer G.L., Henzell R.G. (2000) Does maintaining green leaf area in sorghum improve yield under drought? II. Dry matter production and yield. Crop Science, 40, 1037-1048.

Borrell A.K., Mullet J.E., George-Jaeggli B., van Oosterom E.J., Hammer G.L., Klein P.E., Jordan D.R. (2014) Drought adaptation of stay-green sorghum is associated with canopy development, leaf anatomy, root growth, and water uptake. Journal of Experimental Botany, 65, 6251-6263.

Botha F.C., Black K.G. (2000) Sucrose phosphate synthase and sucrose synthase activity during maturation of internodal tissue in sugarcane. Functional Plant Biology, 27, 81-85.

Chaudhari G.N., Fakrudin B. (2016) Candidate gene prediction and expression profiling of near isogenic lines (NILs) carrying stay-green QTLs in rabi sorghum. Journal of Plant Biochemistry and Biotechnology, doi: 10.1007/s13562-016-0362-x.

Chen D., Wang S., Xiong B., Cao B., Deng X. (2015) Carbon/nitrogen imbalance associated with drought-induced leaf senescence in Sorghum bicolor. PLoS One, 10(8), e0137026.

Duncan R.R., Bockholt A.J., Miller F.R. (1981) Descriptive comparison of senescent and nonsenescent sorghum genotypes. Agronomy Journal, 73, 849-853.

Fu P.L., Jiang Y.J., Wang A.Y., Brodribb T.J., Zhang J.L., Zhu S.D., Cao K.F. (2012) Stem hydraulic traits and leaf water-stress tolerance are co-ordinated with the leaf phenology of angiosperm trees in an Asian tropical dry karst forest. Annals of Botany, 110, 189-199.
Geigenberger P., Reimholz R., Geiger M., Merlo L., Canale V., Stitt M. (1997) Regulation of sucrose and starch metabolism in potato tubers in response to short-term water deficit. Planta, 201, 502-518.

Grof C.P.L., So C.T.E., Perroux J.M., Bonnett G.D., Forrester R.I. (2006) Research Note: The five families of sucrose-phosphate synthase genes in Saccharum spp. are differentially expressed in leaves and stem. Functional Plant Biology, 33, 605-610.

Gutjahr S., Vaksmann M., Dingkuhn M., Thera K., Trouche G., Braconnier S., Luquet D. (2013a) Grain, sugar and biomass accumulation in tropical sorghums. I. Trade-offs and effects of phenological plasticity. Functional Plant Biology, 40, 342-354.

Gutjahr S., Clément-Vidal A., Soutiras A., Sonderegger N., Braconnier S., Dingkuhn M., Luquet D. (2013b) Grain, sugar and biomass accumulation in photoperiod-sensitive sorghums. II. Biochemical processes at internode level and interaction with phenology. Functional Plant Biology, 40, 355-368.

Hoffmann-Thoma G., Hinkel K., Nicolay P., Willenbrink J. (1996) Sucrose accumulation in sweet sorghum stem internodes in relation to growth. Physiologia Plantarum, 97, 277-284. 
Johnson S.M., Cummins I., Lim F.L., Slabas A.R., Knight M.R. (2015) Transcriptomic analysis comparing stay-green and senescent Sorghum bicolor lines identifies a role for proline biosynthesis in the stay-green trait. Journal of Experimental Botany, 66, 7061-7073.

Kakani V.G., Vu J.C., Allen L.H., Boote K.J. (2011) Leaf photosynthesis and carbohydrates of $\mathrm{CO}_{2}$ enriched maize and grain sorghum exposed to a short period of soil water deficit during vegetative development. Journal of Plant Physiology, 168, 21692176.

Kumar C.G., Fatima A., Rao P.S., Reddy B.V., Rathore A., Rao R.N., Khalid S., Kumar A.A., Kamal A. (2010) Characterization of improved sweet sorghum genotypes for biochemical parameters, sugar yield and its attributes at different phenological stages. Sugar Tech, 12, 322-328.

Li X., Su M., Li X., Cheng L., Qi D., Chen S., Liu G. (2014) Molecular characterization and expression patterns of sucrose transport-related genes in sweet sorghum under defoliation. Acta Physiologiae Plantarum, 36, 1251-1259.

Lingle S.E., Dunlap J.R. (1987) Sucrose metabolism in netted muskmelon fruit during development. Plant Physiology, 84, 386-389.

Livak K.J., Schmittgen T.D. (2001) Analysis of relative gene expression data using real-time quantitative PCR and the $2^{-\Delta \Delta C T}$ method. Methods, 25, 402-408.

Mace E.S., Singh V., Van Oosterom E.J., Hammer G.L., Hunt C.H., Jordan D.R. (2012) QTL for nodal root angle in sorghum (Sorghum bicolor L. Moench) colocate with QTL for traits associated with drought adaptation. Theoretical and Applied Genetics, 124, 97-109.
Miller G.L. (1959) Use of dinitrosalicylic acid reagent for determination of reducing sugar. Analytical Chemistry, 31, 426-428.

Murray S.C., Rooney W.L., Mitchell S.E., Sharma A., Klein P.E., Mullet J.E., Kresovich S. (2008) Genetic improvement of sorghum as a biofuel feedstock: II. QTL for stem and leaf structural carbohydrates. Crop Science, 48, 2180-2193.

van Oosterom E.J., Jayachandran R., Bidinger F.R. (1996) Diallel analysis of the stay-green trait and its components in sorghum. Crop Science, 36, 549-555.

van Oosterom E.J., Chapman S.C., Borrell A.K., Broad I.J., Hammer G.L. (2010) Functional dynamics of the nitrogen balance of sorghum. II. Grain filling period. Field Crops Research, 115, 29-38.

Peloewetse E. (2012) Agro-industrial potential of sweet sorghum accessions grown under semi-arid conditions. African Journal of Biotechnology, 11, 1097010975.

Qazi H.A., Paranjpe S., Bhargava S. (2012) Stem sugar accumulation in sweet sorghum -activity and expression of sucrose metabolizing enzymes and sucrose transporters. Journal of Plant Physiology, 169, 605-613.

Qazi H.A., Rao P.S., Kashikar A., Suprasanna P., Bhargava S. (2014) Alterations in stem sugar content and metabolism in sorghum genotypes subjected to drought stress. Functional Plant Biology, 41, 954-962. Schäfer W.E., Rohwer J.M., Botha F.C. (2004) Proteinlevel expression and localization of sucrose synthase in the sugarcane culm. Physiologia Plantarum, 121, 187-195.

Shakoor N., Nair R., Crasta O., Morris G., Feltus A. Kresovich S. (2014) A Sorghum bicolor expression atlas reveals dynamic genotype-specific expression profiles for vegetative tissues of grain, sweet and bioenergy sorghums. BMC Plant Biology, 14, 1.

Subudhi P.K., Rosenow D.T., Nguyen H.T. (2000) Quantitative trait loci for the stay green trait in sorghum (Sorghum bicolor L. Moench): consistency across genetic backgrounds and environments. Theoretical and Applied Genetics, 101, 733-741.

Tari I., Laskay G., Takacs Z., Poor P. (2013) Response of sorghum to abiotic stresses: a review. Journal of Agronomy and Crop Science, 199, 264-274.

Tarpley L., Vietor D.M. (2007) Compartmentation of sucrose during radial transfer in mature sorghum culm. BMC Plant Biology, 7, 1.

Thomas H., Ougham H. (2014) The stay-green trait. Journal of Experimental Botany, 65, 3889-3900.

Tovignan T.K., Fonceka D., Ndoye I., Cisse N., Luquet D. (2016) The sowing date and post-flowering water status affect the sugar and grain production of photoperiodic, sweet sorghum through the regulation of sink size and leaf area dynamics. Field Crops Research, 192, 67-77.

Tyler L., Bragg J.N., Wu J., Yang X., Tuskan G.A., Vogel J.P. (2010) Annotation and comparative analysis of the glycoside hydrolase genes in Brachypodium distachyon. BMC Genomics, 11, 600.

Vadez V., Deshpande S.P., Kholova J., Hammer G.L., Borrell A.K., Talwar H.S., Hash C.T. (2011) Staygreen quantitative trait loci's effects on water extraction, transpiration efficiency and seed yield depend on recipient parent background. Functional Plant Biology, 38, 553-566.

Zegada-Lizarazu W., Monti A. (2013) Photosynthetic response of sweet sorghum to drought and re-watering at different growth stages. Physiologia Plantarum, 149, 56-66. 